\title{
MDT to HIM
}

\author{
Zhiping Yang ${ }^{1}$ and daiming fan ${ }^{2}$ \\ ${ }^{1}$ Shanghai Mingpin Institute of Holistic Integrative Medicine \\ ${ }^{2}$ Fourth Military Medical University
}

October 12, 2020

\begin{abstract}
This is a comment article on MDT, not a original article.
\end{abstract}

\section{MDT to HIM}

Zhiping Yang, $\mathrm{PhD},{ }^{1,2}$ Daiming Fan, $\mathrm{PhD}^{2,3}$

${ }^{1}$ Shanghai Mingpin Institute of Holistic Integrative Medicine, Shanghai, China

${ }^{2}$ China Institute for Development Strategy of Holistic Integrative Medicine, Xi'an, China

${ }^{3}$ State Key Laboratory of Cancer Biology \& National Clinical Research Center for Digestive Diseases, Xijing Hospital of Digestive Diseases, Fourth Military Medical University, Xi'an, China

Correspondence to: Professor Daiming Fan, 127\# West Changle Road, Xi'an 710032, China; Tel, 8629 84771501; Fax, 8629 84771466; E-mail, fandaim@fmmu.edu.cn

Conflict of Interest: None.

Multidisciplinary teams (MDT)s were introduced in 1995 following evidence of variation in cancer care delivery in the UK documented in the Calman-Hine report. ${ }^{1}$ Due to the well-recognized benefits, such as standardization and continuity of care, effective use of resources, improved patient outcomes and safeguarding of patients from maverick doctors, the MDT has become a widely accepted approach for the diagnosis, treatment and management of cancer patients. ${ }^{1,2}$ It has since been extended to many other medical fields involving complex diseases and conditions.

However, the decisions emerging from MDTs have not always been the best options. Dr. Teo et al. described the outcomes of 47 patients who underwent surgical resection despite a previous recommendation by a MDT against surgery. ${ }^{3}$ From their experience, it was clear that the decisions made by the local MDTs were inappropriate and reversing the multidisciplinary decision changed the prognosis for the patients in 32 out of 47 cases.

Why? The development of modern medicine is currently facing big challenges because of the overspecialization and over-division of medical disciplines and the fragmentation of medical knowledge. ${ }^{4}$ Even now the senior professional in the team is only an expert in a very limited field or specialty. Teamwork has increasingly been used to solve this dilemma. As a knowledge team in health care, a MDT is constituted by a group of diverse health professionals, each contributing to the common goal of providing health care in accordance with his/her competence. ${ }^{5}$ All professionals are doing their best within the limitations imposed by current scientific approaches and their own knowledge, but concerted efforts do not necessarily result in positive or optimal healthcare delivery for patients because the expanded procedures do not have any impact 
on survival. Sometimes, it may even lead to harmful outcomes. That is why iatrogenic factors have become the third leading cause for overall mortality in the USA. ${ }^{6}$

More worryingly, MDTs may encourage overtreatment. Collective decision making is known to reduce the sense of individual responsibility and to encourage riskier decisions. Under these circumstances, decisions made collectively in MDTs may be biased towards recommending aggressive treatments that have little positive effect but cause patients much misery. ${ }^{7}$ Who makes the final decision at the MDT meeting? It appears that every clinician in a MDT now fears making clinical decisions. ${ }^{2}$ They consider the MDT to be a defensive tool for escaping personal and legal responsibility in achieving a therapy plan.

In order to improve the working of MDTs, we need two things: a holistic view and integrative thinking. The human body is an organic whole with dynamic changes, and powerful self-regulatory and compensation mechanisms. It has been demonstrated that factors such as failure to consider holistic information or patient's views and lack of personal knowledge of the patient being discussed all had an adverse impact on effective clinical decision making in MDTs. ${ }^{8}$ However holistic thinking looks at the human being as a whole and places patients in a larger context, involving natural, social, psychological, and other factors. The All of Us Research Program (formerly named Precision Medicine Initiative) in fact aims to determine whole health related factors, including lifestyle, socioeconomic factors, environment, and biologic characteristics to advance precision diagnosis, prevention, and treatment by enrolling a large diverse cohort of at least 1 million persons in the USA. ${ }^{9}$

What is integrative thinking? Roger Martin, a famous Canadian management scientist, once elaborated on the concept of integrative thinking: that is, holding two contradictory views in the mind at the same time, and from here he came up with a solution combining the advantages of both perspectives. It aims to deal with opposing views in a constructive way, not at the expense of choosing one over the other, but in an innovative way to eliminate the confrontation between the two views. The new views contain some elements of the opposing points and are superior to both of the original opposing points. ${ }^{10} \mathrm{In}$ order to use integrative thinking it is necessary to grasp several principles: first, expand the scope of the key factors in decisionmaking; Second, consider multi-faceted and indirect causality; Third, in the decision-making process, the problem is not divided between several independent individuals to solve one by one, but to deal with each part while maintaining the integrity of the problem. The fourth is to struggle to find innovative solutions, each idea and process is more efficient and accurate than the previous one. For instance, when an advanced cancer patient is faced with options such as surgery, chemotherapy, radiotherapy, and biological therapy; the order and degree of the treatments, and whether they are to be used in combination or sequentially; this requires integrative thinking to make the final decision in the MDT. This is the Holistic Integrative Medicine (HIM) that we have proposed and advocated. ${ }^{4,11}$

To construct a new medical system more suitable for human health and disease management, HIM regards the human body as a holistic unit; organically integrates the most advanced knowledge and theories in each medical field and the most effective practices from various clinical specialties; and then develops corresponding revisions and adjustments according to social, environmental, and psychological conditions. ${ }^{4}$ From MDT to HIM, we should establish a multidisciplinary working model to formulate an individualized integrated healthcare plan to achieve an optimal effect using the perspective of HIM.

\section{References}

1. Thornton S, Dodwell D. Multidisciplinary team working: the emperor without clothes? $\mathrm{Br} J$ Hosp Med (Lond) 2012;73:186-7.

2. Soukup T, Lamb BW, Sevdalis N, Green JS. Streamlining cancer multidisciplinary team meetings: challenges and solutions. Br J Hosp Med (Lond) 2020;81:1-6.

3. Teo C, Broggi M. Surgical outcome of patients considered to have "inoperable" tumors by specialized pediatric neuro-oncological multidisciplinary teams. Childs Nerv Syst 2010;26:1219-25.

4. Fan D. Holistic integrative medicine: toward a new era of medical advancement. Front Med 2017;11:152-9. 
5. Quinlan E, Robertson S. Mutual understanding in multi-disciplinary primary health care teams. $J$ Interprof Care 2010;24:565-78.

6. Makary MA, Daniel M. Medical error-the third leading cause of death in the US. BMJ 2016;353:i2139.

7. Eigenmann F. Multidisciplinary team meetings encourage overtreatment. BMJ 2015;351:h4630.

8. Soukup T, Lamb BW, Arora S, Darzi A, Sevdalis N, Green JS. Successful strategies in implementing a multidisciplinary team working in the care of patients with cancer: an overview and synthesis of the available literature. J Multidiscip Healthc 2018;11:49-61.

9. All of Us Research Program Investigators, Denny JC, Rutter JL, et al. The "All of Us" Research Program. N Engl J Med 2019;381:668-76.

10. Martin RL. The Opposable Mind: How Successful Leaders Win Through Integrative Thinking. Boston: Harvard Business School Press, 2007: 6.

11. Fan DM. Holistic Integrative Medicine. Am J Digest Dis2014;1:22-36. 